\title{
NONLINEAR DYNAMO OF MAGNETIC FLUCTUATIONS AND FLUX TUBES FORMATION IN THE IONOSPHERE OF VENUS
}

\author{
N. KLEEORIN \\ Department of Mechanical Engineering, Ben-Gurion University \\ of Negev, POB 653, 84105 Beer-Sheva, Israel

\section{ROGACHEVSKII} \\ Racah Institute of Physics, Hebrew University of Jerusalem, \\ 91904 Jerusalem, Israel
}

\section{A. EVIATAR}

Department of Geophysics and Planetary Science, Tel Aviv University, 69978 Ramat Aviv, Israel

ABSTRACT. Magnetic field observations in the dayside ionosphere of Venus revealed the magnetic flux ropes (Russell and Elphic 1979). General properties of these small-scale magnetic field structures can be explained by the theory of magnetic fluctuations excited by random hydrodynamic flows of ionospheric plasma.

A nonlinear theory of the flux tubes formation based on the Zeldovich's mechanism of amplification of the magnetic fluctuations is proposed. A nonlinear equation describing the evolution of the correlation function of the magnetic field can be derived from the induction equation, the nonlinearity being connected with the Hall effect. The large magnetic Reynolds number limit allows an asymptotic study by a modified WKB method.

On the basis of this theory it is possible to explain why the flux tubes are not observed if there is a strong regular large-scale magnetic field when the ionopause is low. The theory predicts the cross section of the flux ropes in the ionosphere of Venus and the maximum value of the magnetic field inside the flux tube.

\section{Introduction}

Filamentary magnetic structures were observed in the ionosphere of Venus (Russell and Elphic 1979). General properties of these smallscale magnetic field structures are following (see, for example, Elphic and Russell 1983, Luhmann 1990): a) the flux tubes are observed only in the regions where average large-scale magnetic field is practically 
zero, b) flux tubes axis inclinations are generally random, c) the magnetic structures stationary while the spacecraft passes through, d) the cross section of the flux ropes is about several tens $\mathrm{km}$.

For explanation of origin of the magnetic flux ropes several mechanisms have been proposed (Wolff et al. 1980, Cloutier et al. 1983, Luhmann and Elphic 1985, Russell et al. 1987). However, the most difficult to understand the formation process of the flux tube in the absence of the large-scale regular magnetic field. Therefore, although the magnetic flux tubes were revealed in the ionosphere of Venus in 1979 , the problem of their origin and evolution still remains the subject of discussions.

In this paper a nonlinear theory of the magnetic flux tubes formation is developed. According to this theory the observed magnetic flux ropes in the ionosphere of Venus can be interpreted as magnetic fluctuations excited by random hydrodynamic flows of ionospheric plasma with zero mean magnetic field. On the basis of this theory we described the general properties of the flux tubes in the ionosphere of Venus.

\section{Magnetic flux ropes}

A mechanism of generation of magnetic fluctuations with zero mean magnetic field was proposed by Zeldovich (see, for example, reviews by Vainshtein and Zeldovich 1972, Zeldovich et al. 1990). An original loop of magnetic field is stretched, twisted and then folded. These non-trivial motions are three-dimensional. They result in an amplification of the magnetic field. Magnetic diffusion leads to reconnection of the field in $\mathrm{X}$-point. If the turbulent flows of conductive fluids tend to two-dimensional, for example, due to an emergence of external field, the magnetic fluctuations are not generated. The appearance of strong regular large-scale magnetic field in the ionosphere of Venus (when the ionopause is low) results in the turbulent flows of ionospheric plasma becomes two-dimensional, and so the flux tubes are not observed.

Investigations by Meneguzzi et al. 1981, Molchanov et al. 1985, Zeldovich et al. 1990 have shown, that the local spatial distribution of the magnetic field is intermittent: the field is concentrated within flux tubes separated by the regions with weak fields. The characteristic lifetime of the turbulent flux tubes is of the order of the turnover 
time of turbulent eddies. In the ionosphere of Venus this time is about several minutes. Therefore in spite of the random character of magnetic fluctuations the spacecraft observations show the stationary picture of flux tubes (see, also Elphic and Russell 1983).

Statistical properties of the turbulent magnetic field $\mathbf{B}$ can be described by the correlation function $W(r, t)=\langle B(\mathbf{x}, t) B(\mathbf{y}, t)\rangle$. Here the angular brackets mean statistical averaging, $B$ is the projection of magnetic field $\mathbf{B}$ on the direction $\mathbf{r}=\mathbf{x}-\mathbf{y}$ (for example, on the direction of the spacecraft path). A nonlinear equation describing the evolution of the correlation function of the magnetic field is close to the Schrödinger equation but features a variable mass and has no imaginary unity in the time-derivative term. The equation takes a simpler form when it is formulated in terms of an auxiliary function $\Psi=r^{2} W / 3 \sqrt{2 m}$. Then it reads

$$
\frac{\partial \Psi}{\partial t}=\frac{1}{m(r, N)} \Psi^{\prime \prime}-U\left(r, N, N^{\prime}, N^{\prime \prime}, \frac{\partial N}{\partial t}\right) \Psi,
$$

where $A^{\prime}$ is the spacial derivative. The nonlinearity is $N=W^{\prime \prime}+$ $4 W^{\prime} / r$. The variable mass $m$ and a potential $U$ are given by

$$
\begin{gathered}
U=\frac{2}{m r^{2}}-\frac{\left(m^{\prime}\right)^{2}}{4 m^{3}}+\frac{\phi^{\prime}}{r}-m \frac{\partial N}{\partial t}, \\
m^{-1}=m_{0}^{-1}(r)+2\left(N-N_{*}\right), \quad m_{0}^{-1}(r)=2 R m^{-1}+\frac{2}{3}(1-F(r)), \\
\phi=\frac{\left(r^{3}(F-N)\right)^{\prime}}{3 r^{2}}, \quad N_{*}=N(r=0) .
\end{gathered}
$$

The function $F(r)$ depends on a correlation function for velocity field (see below). Eq. (1) is written in the dimensionless variables: coordinates and time are measured in the units $l_{0}$ and $l_{0} / u_{0}, l_{0}$ is the main scale of turbulent hydrodynamic pulsations which is of the same order of the density variation scale of neutral gas $\left(l_{0} \simeq(1-10) 10^{6} \mathrm{~cm}\right.$ for ionosphere of Venus), $u_{0}$ is the turbulent velocity $\left(u_{0} \simeq(2-20) 10^{3}\right.$ $\mathrm{cm} / \mathrm{s})$. Magnetic field $B$ and correlation function $W$ are measured in the units $b$ and $b^{2}$, where $b=4 \pi e n l_{0} u_{0} / \sqrt{3} c, e$ is the charge of electron, $n$ is the density of electrons $\left(n \simeq 10^{4}-10^{5} \mathrm{~cm}^{-3}\right)$, c 
is the light speed. The magnetic Reynolds number $R m=u_{0} l_{0} / \eta$ ( $R m \simeq 70-150), \eta=c^{2} / 4 \pi \sigma$ is the magnetic viscosity, $\sigma$ is the electrical conductivity.

The nonlinear term $N(r, \Psi)$ in Eq. (1) is due to the Hall effect. If $N=0$, Eq. (1) is linear. It was derived in different methods by Kazantsev 1967, Vainshtein 1982, Molchanov et al. 1983 for a short-correlated velocity field:

$$
<\mathbf{u}(\mathbf{x}, t) \cdot \mathbf{u}(\mathbf{y}, t+\tau)>=l_{0} u_{0} f(r) \delta(\tau) ; f(r)=\left(r^{3} F\right)^{\prime} / 3 r^{2}=\exp \left(-r^{2}\right),
$$

where $\delta(\tau)$ is the delta function. As follows from analysis by Dittrich et al. (1984) this approximation is valid also for velocity field with finite correlation time if statistical characteristics of magnetic field are changed slow in comparison with the correlation time.

The nonlinear equation (1) can be derived from the induction equation:

$$
\frac{\partial \mathbf{B}}{\partial t}=\operatorname{curl}\left(\mathbf{u} \times \mathbf{B}-\frac{c}{4 \pi e n} \operatorname{curl} \mathbf{B} \times \mathbf{B}+\eta \operatorname{curl} \mathbf{B}\right) .
$$

The second term in Eq. (2) describes the Hall effect. The Eq. (2) is for three-liquid magnetohydrodynamics for electrons, ions and neutral particles with the Om's law:

$$
\mathbf{j}=\sigma\left(\mathbf{E}+\frac{1}{c} \mathbf{u} \times \mathbf{B}+\frac{1}{4 \pi e n} \mathbf{B} \times \operatorname{curl} \mathbf{B}\right), \quad \sigma^{-1}=\frac{m_{e}}{e^{2} n}\left(\tau_{e n}^{-1}+\tau_{e i}^{-1}\right),
$$

where $m_{e}$ is the mass of electron, $\tau_{e n}$ and $\tau_{e i}$ are the times of electronneutral and electron-ion collisions. Note that the nonlinearity $N$ corresponds to a correlation function of the electric current: $N=$ $-3 /\left(e n u_{0}\right)^{2}<j(\mathbf{x}, t) \cdot j(\mathbf{y}, t)>$. Here $j$ is the projection of the electric current $\mathbf{j}$ on the direction $\mathbf{r}=\mathbf{x}-\mathbf{y}$. For the derivation of the nonlinear equation (1) we used method, developed by Molchanov et al. (1983). The main idea of the method consists in the replace of the magnetic diffusion by the Wiener process (Zeldovich et al. 1990). The large magnetic Reynolds number limit allows an asymptotic study by a modified WKB method. Near the threshold of the self-excitation of the magnetic fluctuations the system tends to the steady state for the several correlation times. The threshold of the self-excitation of 
the field in the magnetic Reynolds number is $R m \simeq 67$. The magnitude was found by numerical simulations (Meneguzzi et al. 1981, Novikov et al. 1982) and analytical study (Kleeorin et al. 1990). In the ionosphere of Venus $R m \simeq 70 \div 150$. Thus, we need to find the stationary solution of Eq. (1). The asymptotic solution satisfied the boundary conditions $W(r=0)=W_{0}$ and $W(r=\infty)=0$ is

$$
\frac{W}{W_{0}}= \begin{cases}1 & \text { for } 0 \leq r<R m^{-1 / 2} \\ \frac{R m^{-5 / 4}}{r^{5 / 2}} \cos (\ln (\sqrt{R m} \cdot r)) & \text { for } R m^{-1 / 2} \leq r<R m^{-1 / 4} \\ \frac{R m^{-5 / 4}}{r^{3}} & \text { for } r>1\end{cases}
$$

In the region $0 \leq r<R m^{-1 / 2}$, which corresponds to the magnetic flux tube, the solution $W=W_{0}=$ const coincides with the solution of the linear problem (see Kleeorin et al. 1986, Zeldovich et al. 1990). It means, that the strong nonlinearity ( the large electric current ) can not suppress the generation of the magnetic fluctuations in this region. It seems to be a force-free magnetic field $(\mathbf{j} \times \mathbf{B}=0)$ in the region. So the flux tubes are twisted. In the second region $\left(R m^{-1 / 2}<r<R m^{-1 / 4}\right)$ the function $W$ drastically decreases, the nonlinearity here restricts the level of magnetic fluctuations. The characteristic cross section of the flux tubes is more then $l_{0} \cdot R m^{-1 / 2} \simeq 1 \div 10 \mathrm{~km}$. It is agree with the observation of the flux tubes in the ionosphere of Venus (Russell and Elphic 1979). The numerical stationary solution of Eq. (1) confirms the asymptotic solution (4).

The theory gives the mean square of magnetic field $\left\langle B^{2}\right\rangle$. The magnetic field exist in the form of magnetic ropes separated by the regions with weak magnetic field. After account for that one can get the maximum value of magnetic field inside the flux tube

$$
B_{m} \simeq \frac{4 \pi e n \eta}{c} R m^{3 / 2} \sqrt{W_{0}} \simeq 50-100 n T
$$

\section{References}

Cloutier, P.A., et al., in Venus, eds. D.M. Hunten et al., Univ. Arizona Press, P. 941, 1983. 
Dittrich, P., et al., Astron. Nachr., Bd. 305, Hf. 2, 1984.

Elphic, R.C., and C.T. Russell, J. Geophys. Res., 88, 58, 1983.

Kazantsev, A.P., Sov. Phys. JETP, 26, 1031, 1967.

Kleeorin, N.I., I.V. Rogachevskii, A.A. Ruzmaikin, Intern. Workshop 'Plasma Astrophysics', Abstracts, Telavi, USSR, ESA Spec. Publ., 67, 1990.

Kleeorin, N.I., A.A. Ruzmaikin, D.D.Sokoloff, Proc. Intern. Workshop 'Plasma Astrophysics', Sukhumi, USSR, ESA Spec. Publ., $557,1986$.

Luhmann, J.G. in Geophysical Monograph, American Geophys. Union 58, 425, 1990.

Luhmann, J.G., R.C. Elphic, J. Geophys. Res., 90, 12047, 1985.

Meneguzzi M., U. Frisch, A. Pouquet, Phys. Rev.Lett., 41, 1060, 1981.

Molchanov, S.A., A.A. Ruzmaikin, D.D.Sokoloff, Magnetohydrodynamics, N 4, 67, 1983.

Molchanov, S.A., A.A. Ruzmaikin, D.D.Sokoloff, Sov. Phys. Usp., 28, 307, 1985.

Novikov, V.G., A.A. Ruzmaikin, D.D.Sokoloff, Preprint Keldysh Institute of Applied Mathematics, 138, 1982.

Russell, C.T., R.C. Elphic, Nature, bf 279, 616, 1979.

Russell, C.T., et al., Adv. Space Res., 7, 115, 1987.

Vainshtein, S.I., Sov. Phys. JETP, 56, 86, 1982.

Vainshtein, S.I., Ya.B. Zeldovich, Sov. Phys. JETP, 106, 431, 1972.

Wolff, R.S., B.E. Goldstein, C.M. Yeates, J. Geophys. Res., 85, 7697, 1980.

Zeldovich, Ya.B., A. Ruzmaikin, D.D.Sokoloff, The Almighty Chance, Word Scientific Publ., London, 1990. 